“Transfer” XV: 1-2 (2020), pp. 168-190. ISSN: 1886-554

\title{
EL ENFOQUE FEMINISTA DE LA TRADUCCIÓN E INTERPRETACIÓN: UNA VENTAJA COMPETITIVA
}

María Reimóndez (ORCID: 0000-0002-8775-941X)

$\mathrm{BiFeGa}^{1}$

\section{El enfoque feminista como conflicto}

Seguramente por una cierta distorsión académica, la práctica feminista de la traducción e interpretación haya sido conceptualizada con demasiada frecuencia a través del conflicto y lo excepcional, en vez de a través de un enfoque de lo diario desde lo positivo. El mundo académico, con cierta lógica, tiende a interesarse por aquello que se sale de la norma y que constituye un evento inusual en el marco general de las prácticas. Aunque esto no es negativo en sí mismo, en el caso de la traducción feminista ha generado una visión de embudo que actúa de forma disuasoria ante las profesionales, sobre todo las que comienzan en el mundo laboral.

Si bien en otros campos se analiza el funcionamiento habitual (por ejemplo, en las instituciones, la traducción asistida, médica o legal), en este momento no contamos con estudios que profundicen en el análisis diario de las prácticas traductoras e interpretativas de las profesionales feministas. Carecemos, exceptuando el formato habitual de estudio de alguna traducción en concreto, con frecuencia conflictiva (véanse como ejemplos los incluidos en von Flotow 2001, Kamala 2009, Reimóndez 2009, von Flotow 2011 o Castro \& Ergun 2017), de un proceso más global y complejo de análisis de quiénes

\footnotetext{
${ }^{1}$ BiFeGa, proyecto financiado por la Consellería de Cultura, Educación e Ordenación Universitaria de la Xunta de Galicia en el marco del Programa de Consolidación e Estruturación de Unidades de Investigación Competitivas (2017-2019) con referencia: ED431B 2017176.
} 
“Transfer” XV: 1-2 (2020), pp. 168-190. ISSN: 1886-554

somos, cómo trabajamos y qué estrategias aplicamos las traductoras e intérpretes feministas en el mercado profesional en un lugar, tiempo e idiomas dados.

En realidad, esta afirmación podría extenderse en algunos aspectos al conjunto de la profesión. En el campo literario, por ejemplo, debido a la situación de subalternidad que los estudios feministas de la traducción tan bien han señalado para esta actividad, rara vez se estudia el trabajo de una traductora como obra propia (de las intérpretes, de quien hablaré más adelante, ya nadie ni se lo plantea). La única excepción la constituye el anómalo campo de la llamada "auto-traducción", en el cual la figura del autor (masculino intencional) ejerce todo su poder para generar interés en la actividad traductora. Como estudiosa feminista de la traducción, siempre me ha llamado la atención que ese campo de nuestra disciplina se maraville con las estrategias de Cunqueiro, Navokov y otros, cuando son exactamente las mismas intervenciones que traductoras sin obra propia realizan todos los días (véase Kippur 2015, Rodríguez 2016). La única diferencia es que nadie se ha parado a estudiarlas de forma sistemática a lo largo del tiempo y de las obras (de la traductora).

Si bien es cierto que existen conflictos y dificultades a la hora de ejercer nuestra profesión desde una óptica feminista, también es importante trazar un panorama más complejo y que ayude a abrir otros espacios de reflexión académica. Este panorama tiene que situarse en el espacio, el tiempo y el idioma, en las prácticas concretas y en las teorías situadas para evitar también los habituales sesgos eurocéntricos, blancos y de hegemonía lingüística.

Este artículo busca tratar, desde un marco teórico firme, pero sobre todo desde mi experiencia como intérprete y traductora feminista en el mercado privado gallego, español y portugués principalmente, el enfoque feminista desde lo positivo y entenderlo como una ventaja competitiva en un espacio cambiante y diverso. Para ello, revisaré primero algunos aspectos fundamentales de la profesiona- 
“Transfer” XV: 1-2 (2020), pp. 168-190. ISSN: 1886-554

lidad para pasar luego a explorar la posición feminista como no solo una necesidad ética, sino sobre todo como una necesidad para atender las demandas de un mercado que necesita profesionales competentes.

\section{El concepto de la profesionalidad}

Para poder entender el enfoque feminista de la traducción y de la interpretación como una ventaja competitiva, primero tenemos que desmontar la visión de la profesionalidad que se nos ha transmitido de forma activa y pasiva desde la formación a la vida cotidiana pasando por la investigación académica. Las teorías feministas de la ciencia luego trasladadas a las de la traducción se han esforzado en desmontar los conceptos de "objetividad", "neutralidad" e "imparcialidad" como herramientas que las estructuras de poder patriarcal y colonial tienen para exigir alianzas a sus prioridades y seguirse reproduciendo. Como dice Mona Baker:

Being 'neutral' is of course an illusion of theory; indeed, given the impossibility of being neutral and the nature of power relations, one might ask who the translator or interpreter is expected to be neutral against when they are fed the disciplinary narrative of neutrality. (Baker 2009: 223, énfasis en el original)

Esta visión está lejos de reducirse a una cuestión teórica. En el mundo profesional, seguimos leyendo una y otra vez en contratos, códigos de conducta y debates en foros profesionales que la traductora o intérprete debe ser "fiel" o "neutral" (Angelelli 2003). Se hace necesario, por lo tanto, ir a una raíz más profunda del entendimiento de la profesionalidad.

Las teorías feministas y poscoloniales, en particular la vertiente de los afectos, han demostrado cómo se considera lo racional 
“Transfer” XV: 1-2 (2020), pp. 168-190. ISSN: 1886-554

como masculino y positivo y lo emocional como femenino y negativo (Ahmed 2004, Platero 2012, Vasallo 2018). Así, en la traducción e interpretación, se nos inculca que verse 'afectada' es lo no profesional. Como he argumentado en otros lugares (Reimóndez 2017), sin embargo, la traducción e interpretación son actividades de intervención. Su propia naturaleza consiste en intervenir en los procesos comunicativos entre personas y grupos. En esos procesos, además, las situaciones de desigualdad de poder se articulan a lo largo de muy distintos ejes, desde el género y la raza a, por supuesto, el idioma y el origen. La traducción e interpretación son actividades políticas de toma de decisiones en las que lo emocional, lo ideológico y lo personal están siempre presentes.

Entendidas desde este marco, las teorías de la neutralidad lo único que hacen es desactivar la capacidad consciente de tomar decisiones de las traductoras e intérpretes desde el punto de vista de la ética. Así, llegamos a situaciones como las que describen Emily Apter (2009) y Moira Inghilleri (2009) en relación con las torturas cometidas en la cárcel de Abu Ghraib por el ejército estadounidense a presos iraquís. La sumisión de las y los intérpretes al principio de neutralidad permitió que se cometiesen abusos de todo tipo, como el caso que relata Apter en el que la persona que realizó la interpretación le indicó a un preso que se masturbase delante de la soldado England para que esta no le pegase más (2009: 199). Como bien indica esta teórica, la ATA (American Translators Association), en vez de ofrecer un marco en el que las intérpretes implicadas pudiesen evitar o por lo menos no participar de las torturas, lo promueve con sus conceptos de la neutralidad y fidelidad. Un enfoque político y feminista de la traducción nos diría, como indica Apter, que el papel de las asociaciones profesionales es otro, por ejemplo, el de diseñar

an official policy protecting translators from being enlisted as intermedaries in the cruel and inhuman treatment of prisoners [...], a legal right to desist from translating under circumstances that violate 
“Transfer” XV: 1-2 (2020), pp. 168-190. ISSN: 1886-554

human rights and the Geneva convention on the treatment of prisoners. (Apter 2009: 200)

No es necesario, no obstante, ir a casos que nos pueden parecer extremos, para comprobar cómo la retórica dominante afecta al trabajo de las intérpretes y traductoras en el día a día. De hecho, las ideas de "neutralidad" e "imparcialidad" pretenden marcar una distancia que entra en contradicción con cualquier concepto de profesionalidad en otros campos. Porque si preguntamos en cualquier sector si la implicación o compromiso, eso que en inglés llaman "engagement", es un elemento importante para el trabajo, veremos que de forma mayoritaria se valora como algo positivo. ${ }^{2}$ Resulta paradójico que en nuestro caso se nos insista tanto en mantenernos "al margen" de lo que hacemos, partiendo de la base de que mantenerse al margen de la comunicación siendo traductoras o intérpretes resulta un oxímoron. En el mercado, más bien al contrario, mostrar una implicación en lo que hacemos es lo que marca la diferencia entre buenas y malas profesionales. Una traductora o intérprete feminista, como veremos, presta atención a muchos más aspectos que quien carece de este marco crítico de análisis y ofrece un resultado que la mayoría de clientes valora como indicativo de calidad.

\section{Se buscan traductoras feministas}

Es cierto que tenemos la impresión certera de que el mundo profesional suele ser hostil hacia las traductoras e intérpretes feministas, pero rara vez nos hemos parado a analizar uno de los elemen-

\footnotetext{
${ }^{2}$ Véase solo como ejemplo de muchos otros este artículo publicado en Forbes, no precisamente una revista caracterizada por compromisos feministas y éticos. Disponible en: <<https://bit.ly/2J5qnUp $>>$ (Fecha última consulta 29/5/19)
} 
“Transfer” XV: 1-2 (2020), pp. 168-190. ISSN: 1886-554

tos más relevantes de ese espacio: las y los clientes. Solemos valorar de forma experiencial o académica el mundo profesional a través de las asociaciones o colectivos de traductoras e intérpretes. Cuando la clientela aparece en escena suele hacerlo en forma, como indicaba anteriormente, de conflicto.

Sin duda, la resistencia a las intervenciones feministas en casi cualquier campo es algo demostrado y que está a la orden del día. En el caso de la traducción podemos medirlo ya de principio en la oposición de ciertos sectores al lenguaje no sexista o inclusivo, que de forma constante es demonizado con una importante violencia y animadversión (véase Castro 2008). Conocida es la recalcitrante posura, por ejemplo, de la Real Academia Española al respecto del lenguaje no sexista o inclusivo (2019); postura que no es necesariamente indicativa de todas las demás lenguas peninsulares.

De hecho, en el caso gallego, la Gramática de la Real Academia Galega coordinada por Rosario Álvarez y Xosé Xove (2002) reconoce y da valor al uso inclusivo del lenguaje, señalando que el masculino "extensivo" está dejando de utilizarse como tal. Al hilo de esta cuestión, es importante aquí señalar la importancia de los supuestos márgenes. Resulta a menudo sorprendente la virulencia sexista y racista presente en ciertos espacios que reúnen profesionales de la traducción e interpretación frente a otros, algo que resulta interesante estudiar teniendo en cuenta los idiomas y culturas implicadas, porque en cada una la penetración de la crítica feminista y el entendimiento de la identidad colectiva va a tener una importancia fundamental a la hora de crear un ambiente más o menos proclive al avance del trabajo de las traductoras feministas. En los contextos lingüísticos en los que yo me muevo, llama poderosamente la atención la oposición al cambio en los espacios castellanohablantes. Un caso significativo es el del grupo de Facebook “Traductores e intérpretes" donde sistemáticamente se hacen comentarios de tipo sexista, homófobo y racista, 
“Transfer” XV: 1-2 (2020), pp. 168-190. ISSN: 1886-554

llegando incluso a compartirse el vídeo de Latinxs) $)^{3}$ para insultar a las personas que en él aparecen. Suelen abundar los comentarios y debates que pretenden primar una variedad del castellano (normalmente la de España) sobre las demás y siempre que alguna integrante intenta compartir recursos sobre lenguaje no sexista como importante herramienta profesional, el aluvión de insultos es insólito. Este comportamiento contrasta profundamente con la inquietud que se demuestra en otros grupos por conocer el lenguaje inclusivo o cuestiones que tienen que ver con la identidad de género u orientación sexual a través del lenguaje. ${ }^{4}$

En este contexto, sin embargo, es necesario visibilizar cómo las traductoras e intérpretes feministas promueven sus espacios y hacen presión dentro de ellos, por ejemplo, en las asociaciones, para que estas se vayan transformando. Solo de esta manera se entiende que eventos como el Encuentro Nacional de Estudiantes de Traducción e Interpretación de 2018 haya incluido un panel sobre traducción y feminismo, o en la AGPTI, que desde siempre ha mostrado un compromiso por lo menos formal con el lenguaje inclusivo, haya solicitudes para un curso sobre este tema, que se considera de importancia para la labor profesional.

Podemos, por lo tanto, centrarnos en el conflicto o en el interés. Lo mismo ocurre con la clientela, con la excepción de que la virulencia que encontramos entre las profesionales, por fortuna, en mi experiencia no se ve reflejada en la demanda profesional. Como

${ }^{3}$ Disponible en: <<https://bit.ly/2V8oms7 >> (Fecha última consulta 29/5/19)

${ }_{4}^{4} \mathrm{Si}$ nos mantenemos en el espacio de Facebook, el grupo Medical Translation and Interpreting Crew, compuesto sobre todo por intérpretes en el entorno médico estadounidense que trabajan con población migrante, suele compartir recursos de este tipo. Al ser los dos grupos cerrados resulta imposible compartir sus publicaciones, pero cualquier persona relacionada con la profesión puede acceder a ellos y comprobar las afirmaciones que aquí comparto. 
“Transfer” XV: 1-2 (2020), pp. 168-190. ISSN: 1886-554

veremos a continuación, más bien al contrario. Es por ello muy importante no sobredimensionar los casos conflictivos.

En mi caso, me dedico a la traducción tanto literaria como comercial y, sobre todo, a la interpretación, de la que hablaré más adelante, del inglés y del alemán principalmente al gallego y al castellano. A pesar de lo sonado de mi conflicto con la editorial Rinoceronte (Reimóndez 2009), lo cierto es que este caso ha constituido una excepción y una anomalía que se ha presentado como la normalidad, estigmatizando además un espacio lingüístico, el gallego, que como he sugerido ya, tiene características positivas en cuanto a su flexibilidad ante los discursos críticos de género, en lo que profundizaré a continuación. Por supuesto, la experiencia es específica para este contexto en concreto y puede diferir mucho de otros lugares, pero por lo menos es importante contar con elementos para explorar otros márgenes que la dinámica del pensamiento colonial, racista y de la hegemonía lingüística nos pasa por alto. El hecho de que se estudie sistemáticamente y de forma casi exclusiva aquello que ocurre en los espacios donde interactúan lenguas hegemónicas hace que al final se proyecte la impresión de que en los sistemas lingüísticos no hegemónicos no "pasa nada" o lo que pasa es irrelevante para la colectividad. En realidad, lo cierto es que nuestras situaciones lingüísticas suelen abrir debates de gran complejidad que rara vez son escuchados en el espacio que se denomina "internacional" (y que por desgracia suele ser el de aquellas personas que dominan una lengua hegemónica únicamente). En el caso del gallego, por ejemplo, el hecho de que nuestro sistema editorial se aleje con frecuencia de las prioridades estrictamente comerciales hace que tengamos abiertas las puertas a otras posibilidades de traducción. También, que las traductoras tengamos mayor visibilidad y posibilidad de presentar propues- 
“Transfer” XV: 1-2 (2020), pp. 168-190. ISSN: 1886-554

tas. Sirvan, pues, los ejemplos que doy a continuación, para abrirnos el interés académico por otros lugares y lenguas. ${ }^{5}$

En mi labor como traductora que ha trabajado para casi todas las editoriales en lengua gallega, precisamente lo que se ha valorado como positivo ha sido mi capacidad para ofrecer lecturas feministas de las obras. Muy al contrario de lo que los detractores patriarcales quieren hacernos creer, las intervenciones feministas no consisten necesariamente en "cambiar el género" o reescribir totalmente un texto (que también puede ser), sino que implican una reflexión más en profundidad de las claves de interpretación del mismo. Tal vez nunca nos hayamos parado a pensar qué significa realmente ser una traductora feminista, lejos de aquellos conceptos anglocéntricos del "prefacing, foot-noting, hijacking and supplementing" (LotbinièreHarwood 1991). Para ser traductora feminista una tiene que contar con unas bases teóricas críticas que se manifiesten también de forma consciente en la práctica. Debe estar integrada en los movimientos de pensamiento y práctica feminista de las culturas con las que trabaja, conocer sus prioridades, debates y lenguajes y tener la capacidad para desarrollar estrategias de negociación que tengan un impacto en el sistema. Las traductoras e intérpretes feministas, igual que cualquier otra profesional que trabaje desde este marco ético, entiende su función dentro de lo colectivo, dentro de un proyecto de transformación social más amplio y toma decisiones reflexionando sobre cómo lo que hace ayuda a reafirmar o subvertir los valores patriarcales dominantes. Porque lo cierto es que con demasiada frecuencia es la traducción la que introduce sesgos sexistas no presentes en el texto original, como veremos luego, y no solo de textos feministas donde la violencia ha sido tradicionalmente bastante extrema.

\footnotetext{
${ }^{5}$ Pienso por ejemplo en lo lamentable que resulta que nadie haya estudiado nunca el trabajo ingente de traducción feminista que realizan las editoriales indias Zubaan (en: $<<$ https://zubaanbooks.com/>>), y Kali for Women (en: <<https://openlibrary.org/ publishers/Kali_for_Women $>>$ ) (Fecha última consulta 29/5/19).
} 
“Transfer” XV: 1-2 (2020), pp. 168-190. ISSN: 1886-554

Las editoras y editores con los que he trabajado en Galicia han valorado siempre esta capacidad para producir un texto donde no se añadan lecturas machistas, como en el caso de la adaptación de $O s$ tres mosqueteiros (Rivière \& André 2017) o A miña familia (Bottin En prensa) de las editoriales Baía Edicións y Alvarellos respectivamente. ${ }^{6}$ Así, en el primer caso, informé a Baía Edicións de la problemática adaptación del texto que me presentaban como original y les propuse cambios que no solo resultaban más certeros en cuanto a la adaptación del texto de Dumas (que, lejos de ser una historia de "amistad", constituye más bien la bajada a los infiernos de un idealista), sino que daban a las niñas y niños de hoy claves para entender a los personajes femeninos en su contexto, y elementos para ver de forma crítica la violencia que se ejercía contra algunos de ellos. En el caso de $\mathrm{Ma}$ famille fue el propio editor quien me solicitó que revisase algunos aspectos problemáticos del texto relacionados con la racialidad (por ejemplo, mencionar "África" sin determinar el país del que se hablaba en concreto, como si todo el continente fuera un único lugar) o algunas referencias a las relaciones familiares que reforzaban una visión heteropatriarcal de las mismas.

Igual que en el caso de Alvarellos, también la editora Andrea Jamardo y David Cortizo de Urco me propusieron la traducción de $A$ cámara do sangue, de Angela Carter (2018) de forma específica porque en este caso valoraron que solo una traductora feminista podía enfrentarse a este texto y conseguir que encontrase su público en gallego.

Estos casos ilustran como los procesos de intervención feministas empiezan antes de traducir el propio texto, con la selección de lo que se traduce o en procesos de diálogo con los demás agentes

\footnotetext{
${ }^{6}$ Ni Baía Edicións ni Alvarellos se consideran editoriales feministas. Baía sí ha mostrado un mayor compromiso con la publicación de textos para la igualdad, pero ambas son editoriales sin una marca concreta, pero que comprenden que los valores sexistas deben quedar fuera de lo que publican como práctica general.
} 
“Transfer” XV: 1-2 (2020), pp. 168-190. ISSN: 1886-554

implicados. Algo fundamental para ejercer la traducción desde el feminismo y que solo la teoría feminista nos enseña es precisamente el poder de negociar. Cuando hablamos de empoderar a la traductora, esto no se resume a un ejercicio abstracto, sino a la posibilidad de hacer valer lo que consideramos correcto en un proceso de diálogo de igual a igual con los demás agentes implicados en la traducción, principalmente con las editoriales en el caso de la traducción literaria. Este proceso es fundamental también en otro tipo de traducciones y marca la diferencia entre aquellas personas que ofrecen un trabajo mediocre y las que ofrecen un trabajo excelente. Mi experiencia fuera del ámbito literario coincide con la de Daniela Beuren (2001), el único caso documentado que he encontrado entre las lenguas que conozco que narre cómo en el mercado privado no literario (alemán en su caso), la explicación de sus principios éticos (no traducir textos de abierto contenido sexista, racista, fascista/nazi o de la industria del armamento) mejoró su posición comercial. Su clientela entendía en esta postura una reflexión y preocupación por la calidad del trabajo, además de que se transmitía así una "especialización” para cierto tipo de textos. En mi caso, esto también ocurre con frecuencia, por ejemplo, en la traducción médica del inglés al gallego, donde a menudo me encuentro con textos que, a pesar de haber pasado por una traductora y una revisora, llegan a mí en la fase de control de calidad dirigiéndose a mujeres embarazadas en masculino, o aquellos donde la clara distinción del inglés entre "patients", "male/female patients" queda borrosa por el uso impreciso del masculino como aglutinador. Cuando una traductora decide traducir "patients" por "los pacientes", resulta sumamente confuso distinguir cuándo se habla del grupo total y cuándo solo de hombres. Este tipo de cuestiones no son en absoluto menores y la clientela valora que se le informe de este tipo de problemas, o de cómo el uso escrupuloso que algunas empresas hacen del lenguaje inclusivo en inglés desaparece de forma sistemática en su traducción al gallego o castellano, a menos que haya una 
“Transfer” XV: 1-2 (2020), pp. 168-190. ISSN: 1886-554

traductora que interrumpa ese proceso. Porque está claro que para muchas empresas tratar a las personas a las que se dirigen de forma respetuosa e inclusiva en cuanto al género es una prioridad que supera con creces lo puramente lingüístico. El argumento de que en las lenguas romances resulta más engorroso utilizar lenguaje inclusivo no se sostiene ni de lejos, pues igual que en nuestro caso tenemos que concordar la adjetivación y otros elementos, en inglés resulta imprescindible marcar los posesivos, por ejemplo, o los sujetos. Ambas lenguas han desarrollado, gracias a los esfuerzos de las lingüistas feministas, estrategias apropiadas para este tipo de textos. La cuestión es, por lo tanto, si la traductora tiene un conocimiento experto de este aspecto de su profesión, si se ha preocupado por desarrollar sus propias estrategias o simplemente se deja llevar por la visión patriarcal dominante que, como ya he comentado, busca que no pensemos y solo reproduzcamos las estructuras de opresión en las que hemos sido socializadas.

Así, tanto en el mundo editorial como en el mercado de otro tipo de textos, las y los clientes buscan activamente traductoras que se esfuercen por crear textos donde los marcos heteropatriarcales no se reproduzcan. Tras los desastres del pasado, el mundo editorial, por lo menos el gallego, ha reflexionado sobre la importancia de la traducción desde una óptica feminista. Igualmente el mundo empresarial busca llegar a sus destinatarias desde una base que parta del respeto a la igualdad y a las identidades de género inclusivas y diversas.

Aquí vemos también cómo cuando en ciertos espacios se habla del divorcio entre la teoría y la práctica en nuestro campo, en el caso de la traducción feminista la teoría y la práctica han ido de la mano. Nuestro trabajo del día a día solo es posible gracias a los esfuerzos que se han realizado desde el mundo académico, que han contribuido a revelar, por ejemplo, cómo las obras de las autoras feministas sufren procesos intensos de violencia en la traducción (véase la 
“Transfer” XV: 1-2 (2020), pp. 168-190. ISSN: 1886-554

recopilación de ejemplos que incluyo en Reimóndez 2015). Si bien esto pasaba desapercibido en el pasado, en muchos espacios ha dejado de ser así. La difusión social de la investigación ha llevado también a que las lectoras feministas sean más críticas a este respecto y muestren un rechazo a productos que en su traducción transforman elementos transgresores del original en elementos patriarcales, como por ejemplo con la traducción de títulos como We Should All Be Feminists de Chimamanda Ngozi Adichie (2014) al gallego por Todos teriamos que ser feministas (2017, en traducción de Moisés Barcia) y al castellano por Todos deberíamos ser feministas (2015, en traducción de Javier Calvo). Desde el ámbito feminista, incluso aquellas lectoras que no acceden al original son capaces de detectar incoherencias que a esta altura señalan más a la traducción que a la autora original (Aríns 2017). Finalmente, comprobamos cómo los nuevos proyectos editoriales que aparecen alrededor de la edición feminista, buscan de forma activa traductoras que sean capaces de poner en contacto a las autoras con sus lectoras de una forma crítica y harmónica. Un ejemplo de ello es la aparición reciente de la editorial española Ménades que cuenta, entre otras, con la traductora feminista Carla Bateller Estruch. $^{7}$

\section{¿Interpretación feminista?}

Hemos visto hasta ahora que el campo de la traducción, el pensamiento y práctica feministas han ido dando pasos positivos. Esta situación contrasta con el silencio que rodea la interpretación en todas sus modalidades. Como han manifestado los estudios de la interpretación, evaluar la práctica de las intérpretes resulta complejo por cuestiones intrínsecas a la actividad (Cronin 2002). Desde su

${ }^{7}$ En: <<https://menadeseditorial.com/>> (Fecha última consulta 29/5/19). 
“Transfer” XV: 1-2 (2020), pp. 168-190. ISSN: 1886-554

carácter oral hasta la estricta confidencialidad de ciertos encargos, investigar de forma empírica lo que las intérpretes hacemos resulta complicado. Sin embargo, podemos encontrar otras vías, empezando por algunas que ya se han explorado con profusión para las traductoras como las autoevaluaciones o los grupos de debate. Hasta ahora, los Estudios de Interpretación han utilizado todas estas herramientas, pero nunca para enfocarlas desde la óptica feminista (véanse los ejemplos de Angelelli 2003, Martin \& Ortega Herráez 2009, Mason 2009, entre otros). A falta de un estudio más detallado desde este enfoque, me ceñiré a mi experiencia profesional de más de veinte años para explorar algunos aspectos relativos a la importancia del enfoque feminista en la interpretación.

Seguramente, la interpretación en todas sus modalidades sea el espacio donde más desigualdad de poder se pueda palpar en los intercambios comunicativos. Sea interpretando en los juzgados o comisarías o en el ambiente más aparentemente aséptico de los congresos, las intérpretes median siempre en situaciones en las que alguna de las partes está a merced de la otra, donde a veces la brecha de poder es tan poderosa que puede marcar la diferencia entre la libertad o la prisión, entre la salud o la enfermedad, entre la colaboración o el conflicto.

Las estrategias lingüísticas de las intérpretes, además, están restringidas por las dificultades del propio tiempo para interpretar. Mientras que una traducción es un trabajo en el que se escribe, reflexiona y revisa, en la interpretación en cualquiera de sus modalidades (de forma extrema en la simultánea) las decisiones se toman en cuestión de segundos. Es por lo tanto más importante que nunca tener algún tipo de brújula y suficientes estrategias para salir al paso de cuestiones éticas de diversa índole. Aquí es más que en ningún otro lugar donde la profesionalidad como intervención consciente marca la diferencia. 
“Transfer” XV: 1-2 (2020), pp. 168-190. ISSN: 1886-554

El uso de lenguaje no sexista resulta fundamental en la interpretación, pero lo es más todavía cuando se está ante colectivos mayoritarios de mujeres, por ejemplo. O bien cuando el tema es la igualdad o semejantes. En numerosas ocasiones, participantes de congresos de diversa índole se han acercado a la cabina a felicitar el trabajo de interpretar con lenguaje inclusivo, porque no les pasa desapercibido. En mi combinación más habitual, del inglés a lenguas romances y viceversa, está claro que la mayoría de oradoras originales en inglés no determinan el género de muchas cosas. Con frecuencia se refieren a "doctor", "Minister", "Officer", "nurse" o "friend" sin que sepamos el género de esa persona inicialmente o ni siquiera más adelante (por ejemplo, en afirmaciones frecuentes como: "Dr X could not be with us today"). Es necesario tomar decisiones, que por desgracia suelen ir en el sentido de doctor $=$ doctor, pero nurse $=$ enfermera. En mi caso, ante la duda, opto por el femenino, igual de válido que el masculino y con mucha frecuencia, correcto, dado que hoy en día, en nuestro contexto social, son muchas las doctoras, ingenieras y Ministras que salen a colación. Por desgracia, a pesar del incremento de la presencia de mujeres en una gran cantidad de sectores profesionales, con demasiada frecuencia siguen siendo los hombres los que dominan las mesas redondas o el lugar del formador mientras que las mujeres ocupan el del público o alumnado. En estos contextos no resulta inusual escuchar de forma sistemática comentarios de corte sexista, algunos bastante gruesos. Muchas intérpretes se sienten incomodadas ante esto y omiten, igual que omitimos a veces el manido "debe haber habido un error de traducción" cuando dos personas no se entienden. En mi caso, sin embargo, interpreto también las aberraciones para que las presentes puedan protestar, como pasa con más frecuencia de la que parece. Imaginemos un experto en economía que habla de cómo atraer inversión extranjera es como llevarse a una mujer a la cama, "te la quieres follar, pero antes tienes que llevarla a cenar"; o el coloproctólogo que se dedica en toda su 
“Transfer” XV: 1-2 (2020), pp. 168-190. ISSN: 1886-554

presentación a poner fotos de glúteos de mujeres jóvenes con chascarrillos de tipo sexual, o aquel que en un congreso sobre lactancia afirma que las mujeres y crías sobrevivieron gracias a la protección de los hombres. Los ejemplos son tan numerosos como los congresos. Al interpretar la aberración, les damos a las presentes la potestad de protestar o rebatir. Nosotras mismas podemos también hacerlo ante la organización, si nos parece procedente o productivo (por ejemplo, porque quien organiza no estaba presente durante la presentación y no sabe lo que el orador ha comentado y puede considerar no volver a invitar a alguien que insulta al 50\% o más del público presente).

Las intérpretes necesitamos desarrollar estrategias que se puedan utilizar cuando llega el momento. Sabemos que, en las consecutivas, una estrategia importante puede ser distanciarse del orador, pero desde un punto de vista feminista, tal y como indican las estudiosas de la interpretación en contextos de violencia de género como el grupo SOS-VIC, a veces es importante mantener la primera persona cuando estamos transmitiendo el testimonio de una mujer que ha sido víctima de violencia. La guía de la Glasgow Violence Against Women Partnership para intérpretes recomienda esta estrategia, además de entender que las intérpretes son partes ideológicas del proceso. Este es uno de los pocos documentos profesionales donde esto ocurre y donde también se incide en la necesidad de ofrecer un trato correcto a las víctimas (no "neutral", sino ético) y se especifican de forma muy clara los límites para realizar este trabajo. Está claro que en contextos de este tipo un enfoque feminista de la profesión marca la diferencia entre ofrecer el apoyo a quien lo necesita o trabajar para que el sistema pueda revictimizar a las mujeres.

En general, como demuestra el contexto concreto de la interpretación con víctimas de violencia de género, la interpretación desde un enfoque feminista nos ayuda a entendernos como personas que mediamos en un entorno de frágil acceso a los derechos. Desde el 
“Transfer” XV: 1-2 (2020), pp. 168-190. ISSN: 1886-554

respeto a las mujeres o personas LGTBI en los entornos médicos (véase en LGBTHEALTHEDUCATION la guía para intérpretes como ejemplo) hasta la preocupación por la desigualdad en la participación en los congresos forman parte de un entendimiento feminista de nuestro trabajo. Porque las intérpretes estamos ahí para velar para que ciertas personas tengan acceso a la palabra, que en el fondo es que tengan acceso a sus derechos.

\section{Conclusiones}

Explorar las posibilidades de la traducción e interpretación feminista como forma de mejorar el rendimiento profesional de las traductoras e intérpretes resulta fundamental desde todos los puntos de vista. El desarrollo de estrategias conscientes, como hemos visto, es vital para poder ejercer la profesión de una manera responsable y consciente. Cuando no se valora el enfoque feminista como una ventaja competitiva, tampoco se ofrecen herramientas para poder ejercerla. Igual que en la formación reglada se instauran una serie de principios teóricos, también en la práctica se ofrecen al alumnado herramientas o la posibilidad de desarrollarlas. Mientras la traducción e interpretación feminista no se vea como fundamental para trabajar en el mercado, se les negará a las futuras profesionales (y a aquellas que trabajan ya como tales) esta posibilidad. Como indica el estudio de Corrius et al. sobre la sensibilidad de género al traducir audiovisual entre alumnado de grado:

Students rejected gender stereotypes present in contemporary advertising; on the other, they were in favor of using non-sexist language but used it inconsistently in their own translation tasks. (Corrius et al. 2017: 120) 
“Transfer” XV: 1-2 (2020), pp. 168-190. ISSN: 1886-554

Es importante, a partir de la base teórica existente, que el mundo académico se interese de forma más sistemática por la práctica y nos ayude a construir un puente todavía más sólido por el que vayan pasando cada vez más traductoras e intérpretes conscientes, éticas y profundamente profesionales. En resumen, más traductoras e intérpretes feministas. Porque en el fondo, ser buena profesional parte de ser una persona comprometida con la justicia. Como confirma Maria Tymoczko:

the injunction to be "neutral" eviscerates the agency of translators; approaches to the ethics of translation recommending neutrality are paradigmatic of the dispersal of ideology in translation studies and the tendency to efface ethical issues in translation training. [...] One form that the dispersal of ethics, ideology and translator agency takes is the restriction of the focus of professional ethics to the micro levels of textual fidelity and immediate obligations to the employer, effacing larger spheres of responsibility to communities and the world. (2009: 186)

En los lugares donde el poder (de género, clase, origen, lugar...) se toca, las traductoras e intérpretes ocupamos un lugar privilegiado. Es hora de que tomemos postura para que ese privilegio nos ayude a forjar un mundo más justo para todas. Porque empoderarse es reconocer nuestro poder para transformarlo o dejarlo como está.

\section{Referencias bibliográficas}

ADICHIE, Chimamanda Ngozi. (2014). We Should All Be Feminists. Nueva York: Random House.

---. (2015). Todos tendríamos que ser feministas. Barcelona: Random House. Traducción de Javier Calvo. 
“Transfer” XV: 1-2 (2020), pp. 168-190. ISSN: 1886-554

---. (2017). Todos teriamos que ser feministas. Cangas: Rinoceronte Editora. Traducción de Moisés Barcia.

AHMED, Sara. (2004). The Cultural Politics of Emotion. Edimburgo: Edinbourg University Press.

ÁLVAREZ, Rosario \& XOVE, Xosé. (2002). Gramática da lingua galega. Vigo: Galaxia.

ANGELELLI, Claudia. (2003). "The Visible Co-Participant: The Interpreter's Roles in Doctor-Patient Encounters". En: METZGER, Melanie; COLLINS, Steven \& DIVELY, Valerie. (eds.) From Topic Boundaries to Omission: New Research on Interpretation. Washington: Gallaudet University Press, pp. 3-26.

APTER, Emily. (2009). "Translation 9/11: Terrorism, inmigration, language politics". En: BIELSA, Esperanza \& HUGHES, Christopher W. (eds.). Globalization, political violence and translation. Basingstoke: Palgrave Macmillan, pp. 196-206.

ARÍNS, Susana. (2017). "Todas as pessoas teríamos que ser feministas, mesmo as tradutoras". A Sega, Agosto 2017. Disponible en: $<<$ https://bit.ly/2PVNjpI $>>$ (Fecha última consulta 29/5/19).

BAKER, Mona. (2009). "Resisiting state terror: Theorizing communities of activist translators and interpreters". En: BIELSA, E. \& HUGHES, Christopher W. (eds.). Globalization, political violence and translation. Basingstoke: Palgrave Macmillan, pp. 222-242.

BEUREN, Daniela. (2001). "Mein traslatorisch-feministischer Alltag". En: MESSNER, Susane \& WOLF, Maria. (eds.). Graz: Leykam, pp. 31-33.

BOTTIN, Isha. (En prensa). A miña familia. Santiago de Compostela: Alvarellos Editora. Traducción de María Reimóndez con supervisión de Ana Mirás.

CASTRO, Olga. (2008). "Rebatendo o que outros din da linguaxe non sexista”. Festa Da Palabra Silenciada (24): 39-49. 
“Transfer” XV: 1-2 (2020), pp. 168-190. ISSN: 1886-554

CASTRO, Olga \& ERGUN, Emek. (2017). Feminist Translation Studies. Local and Transnational Perspectives. Nueva York-Londres: Routledge.

CARTER, Angela. (2018). A cámara do sangue. Santiago de Compostela: Urco Editora.

CORRIUS, Montse; ESPASA, Eva. (2017). "Balancing Gender Awareness and Professional Priorities in Advertising and Audiovisual Translation: An Overview". En: SANTAEMILIA, José. (ed.) Traducir para la igualdad sexual. Granada: Editorial Comares, pp. 109121.

CRONIN, Michael. (2002). "The empire talks back: Orality, heteronomy and the cultural turn in Interpretation Studies”. En: TYMOCZKO, Maria \& GENTZLER, Edwin. (Eds.). Translation and power. Amherst: University of Massachusetts Press, pp. 45-62.

FLOTOW, Luise von. (2001). “Gender Konzepte im Wandel. Übersetzungspolitische”. En : MESSNER, Sabine \& WOLF, Michaela (eds.). Übersetzung aus aller Frauenländer. Graz: Leykam, pp. 49-59 .

---. (2011). Translating women. Ottawa: University of Ottawa Press.

INGHILLERI, Moira. (2009). "Translators in war zones". En: BIELSA, Esperanza \& Hughes C.W. (eds.). Globalization, political violence and translation. Basingstoke: Palgrave Macmillan, pp. 207-221.

KAMALA, N. (2009). Translating women: Indian Interventions. New Delhi: Zubaan.

KIPPUR, Sara. (2015). Writing It Twice: Self-Translation and the Making of a World Literature in French. Evanston: Northwestern University Press.

LOTBINIËRE-HARWOOD, Suzanne. (1991). Re-belle et infidèle: La traduction comme pratique de réécriture au féminin $=$ The body bilingual: Translation as a re-writing in the feminine. Montréal: Éditions du Remue-ménage.

MARTIN, Anne \& ORTEGA HERRÁEZ, Juan Miguel. (2009). "Court Interpreters' Self Perception”. En: RICOY, Raquel; PÉREZ, Pedro \& 
“Transfer” XV: 1-2 (2020), pp. 168-190. ISSN: 1886-554

WILSON, Christine. (eds). Interpreting and Translating in Public Service Settings. Manchester: St. Jerome Publishing, pp. 141-155.

MASON, Ian. (2009). "Role, Positioning and Discourse in Face-to-Face Interpreting”. En: RICOY, Raquel; PÉREZ, Pedro \& WILSON, Christine. (eds). Interpreting and Translating in Public Service Settings. Manchester: St. Jerome Publishing, pp. 52-73.

LGBTHEALTHEDUCATION. (sd). Glosario de términos LGBT para equipos de atención a la salud. Disponible en: $<<$ https://bit.ly/2LqnsaC>> (Fecha última consulta 29/5/19).

PLATERO, Lucas R. (2012). “Introducción”. En PLATERO, Lucas R. (ed.). Intersecciones: Cuerpos y sexualidades en la encrucijada: Temas contemporáneos. Barcelona: Bellaterra, pp. 15-72.

RAE (Real Academia Española). "Los ciudadanos y las ciudadanas, los niños y las niñas". Disponible en: <<https://bit.ly/1lyCUgW $>>$. (Fecha última consulta 29/5/19).

REIMÓNDEZ, María. (2009). "The curious incident of feminist translation in Galicia: Courtcases, lies and gendern@tions".Galicia 21 Journal of Contemporary Galician Studies, (A): 68-89.

---. (2015). Teorías e prácticas da tradución feminista e poscolonial no contexto galego: un percorrido pola ideoloxía”. Vigo: Universidade de Vigo.

---. (2017) "Distance or Engagement? Questioning Mainstream Discourses on Interpreter Professionalism from a Feminist and Postcolonial Perspective". En: SANTAEMILIA, José. (ed.) Traducir para la igualdad sexual. Editorial Comares, pp. 137-148.

RIVIÈRE, Bénédicte \& ANDRÉ, Camille. (2017). Os tres mosqueteiros. A Coruña: Baía Edicións. Traducción de María Reimóndez.

RODRÍGUEZ VEGA, Rexina. (2016). "Panorama de la autotraducción en Galicia. La especificidad del trasvase entre lenguas en contacto en contexto diglósico". Pasavento. Revista de Estudios Hispánicos, IV (2, verano): 329-346. 
“Transfer” XV: 1-2 (2020), pp. 168-190. ISSN: 1886-554

TYMOCZKO, Maria. (2009). "Translation, ethics and ideology in a violent globalizing world". En: BIELSA, Esperanza \& Hughes Christopher W. (eds.). Globalization, political violence and translation. Basingstoke: Palgrave Macmillan, pp. 171-194.

TRADUCTORES E INTÉRPRETES (2019). Disponible en: $<<$ https://www.facebook.com/groups/interpretes.traductores/>> (Fecha última consulta 29/5/19).

VASALLO, Brigitte. (2018). Pensamiento monógamo. Terror poliamoroso. Madrid: La oveja roja.

Fecha de recepción: 2.05.2019 Fecha de aceptación: 01.06.2019

\section{Resumen:}

Tradicionalmente, la investigación nos ha presentado la práctica de la traducción feminista como un espacio de conflicto, algo que no anima a jóvenes profesionales a trabajar desde esta perspectiva ética. Si bien es cierto que un enfoque feminista ante la vida implica un cierto nivel de oposición y conflicto, también es cierto que la parte positiva de ser una traductora feminista no ha sido estudiada y por lo tanto permanece invisible. Este artículo se centra en las ventajas de ser traductora e intérprete feminista en el mercado y cómo este enfoque es fundamental para una labor realmente profesional.

Palabras clave: Traducción feminista, Interpretación feminista, Profesionalidad 
“Transfer” XV: 1-2 (2020), pp. 168-190. ISSN: 1886-554

\title{
FEMINIST TRANSLATION AND INTERPRETING - A COMPETITIVE ADVANTAGE
}

\begin{abstract}
:
The literature has traditionally presented feminist translation as a space for conflict. Most young professionals feel discouraged to work from this ethical perspective. While it is true that feminist approaches to life entail a certain degree of opposition and strife, it is also true that the positive side of being a feminist translator has often been left unstudied, and therefore in the dark. This article looks at the advantages of working as a feminist translator and interpreter in the market, and how this approach is key to a deeper understanding of professionalism.
\end{abstract}

Keywords: Feminist Translation, Feminist Interpreting, Professionalism. 